\title{
Recurrence entropy and financial crashes
}

\author{
Vladimir Soloviev* \\ Department of Computer Science and \\ Applied Mathematics \\ State Pedagogical University \\ Kryvyi Rih, Ukraine \\ vnsoloviev2016@gmail.com
}

\author{
Olexandr Serdiuk \\ Department of Applied Mathematics \\ Bohdan Khmelnitsky National \\ University of Cherkasy \\ Cherkassy, Ukraine \\ serdyuk@ukr.net \\ Oksana Kohut-Ferens \\ Department of International Ekonomic \\ Ralations \\ Vasyl Stefanyk National Univesity \\ Ivano-Frankivsk, Ukraine \\ ferenso@cloud.com \\ https://orcid.org/ 0000-0003-1888-8397
}

\author{
Serhiy Semerikov \\ Department of Computer Science and \\ Applied Mathematics \\ State Pedagogical University \\ Kryvyi Rih, Ukraine \\ semerikov@gmail.com
}

\begin{abstract}
Entropy is one of the most frequently and effectively used measure of the complexity of systems of various nature. And if the Shannon's canonical entropy is more a measure of the randomness of the system, then the approximate, sample, permutation and other new type entropy that have appeared recently, exploiting the Shannon entropy form have allowed us to quantify the complexity of the systems in question using fast and efficient algorithms. For the first time, a new type of recurrence entropy is used to analyze the dynamics of financial time series under crashes conditions. It is shown that recurrent entropy can be used as the indicatorpredictor of financial crashes.
\end{abstract}

Keywords-recurrence plot, recurrence quantification analysis, recurrence entropy.

\section{INTRODUCTION}

During last few decades the behavior of global financial system attracted considerable attention. Wild fluctuations in stock prices lead to sudden trend switches in a number of stocks and continue to have a huge impact on the world economy causing the instability in it with regard to normal and natural disturbances [1]. The reason of this problem is the crisis of methodology modeling, forecasting and interpretation of socio-economic realities. The doctrine of the unity of the scientific method states that for the study of events in socio-economic systems, the same methods and criteria as those used in the study of natural phenomena are applicable. Similar idea has attracted considerable attention by the community from different branches of science in recent years [2].

The increasing mathematical knowledge of the complex structures of nonlinear systems has provided successful tools to the understanding of irregular space and temporal behaviors displayed by collected data in all applied sciences. Time series analysis has turned to be a key issue providing the most direct link between nonlinear dynamics and the real world [3]. Among the many methods of analysis of complex nonlinear, non-stationary emergent signals, which are the signals of complex systems, those that adequately reflect the spatial and temporal manifestations of complexity are especially popular [4]. In this case, the search for such quantitative measures of complexity that adequately reflect the dynamics of processes taking place in a complex system is relevant. Financial systems being complex dynamic objects exhibit unexpected critical phenomena, which are most clearly manifested in the form of crashes. Over the past 20 years, these are the global currency crisis of 1998, the collapse of the dotcoms 2001, the global financial crisis of 2008, the European debt crisis of 2012, the Chinese crisis of 2015-2016 and the crisis of the US stock market in early 2019 [5]. For this reason, it is extremely important to highlight such measures of complexity that are sensitive to critical phenomena and can serve as their predictors $[6,8]$.

In this paper, we will consider the possibilities of new entropy indicators of the systems complexity, calculated in the phase space, and examine their capabilities with respect to the prevention of crisis phenomena.

\section{THE RECURRENCE BASED ENTROPY}

In recent years, new quantifiers of nonlinear time series analysis have appeared based on properties of phasespace recurrences [7]. According to stochastic extensions to Taken's embedding theorems the embedding of a time series in phase space can be carried out by forming time-delayed vectors $\vec{X}_{n}=\left[x_{n}, x_{n+\tau}, x_{n+2 \tau}, \ldots, x_{n+(M-1) \tau}\right]$ for each value $x_{n}$ in the time series, where $M$ is the embedding dimension, and $\tau$ is the embedding delay. These parameters are obtained by systematic search for the optimal set. Figure 1 shows a phase portrait of the normalized logarithmic returns of the time series of bitcoin (BTC) prices for the period July 17, 2010 to August 30, 2019.

A modern visualization method known as recurrence plot (RP), and is constructed from the recurrence matrix $\vec{R}_{i j}$ defined as $\vec{R}_{i j}(\varepsilon)=\Theta\left(\varepsilon-\left\|x_{i}-x_{j}\right\|\right), x_{i} \in \square, i, j=1,2, \ldots, M$, where $x_{i}$ and $x_{j}$ represent the dynamical state at time $i$ and $j, \Theta$ is the Heaviside function, $M$ is length of theanalyzed time series and $\varepsilon$ is the threshold or vicinity parameter, consisting of a maximum distance between two points in a trajectory such that both points can be considered recurrent to each other. 
The recurrence plot for the phase portrait of Figure 1 is presented in Figure 2.

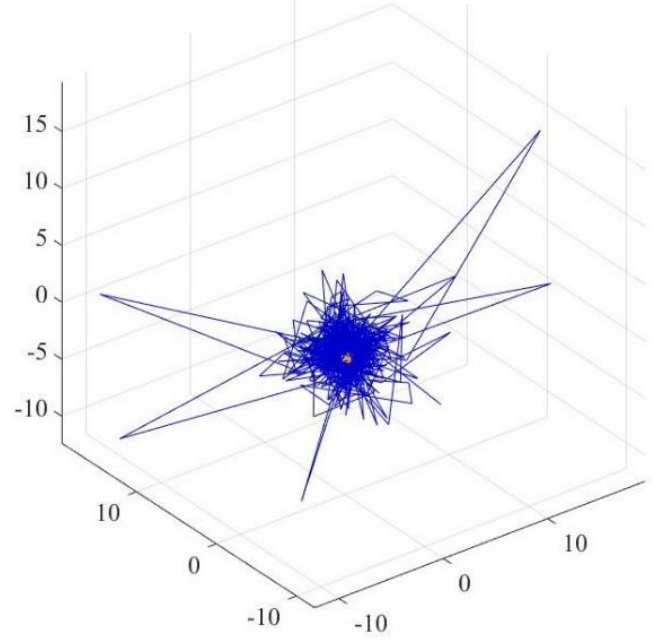

Fig. 1. A phase portrait of the normalized logarithmic returns of the daily values BTC/\$ for the period July 17, 2010 to August 30, 2019.

The graphical representation of the RP allows to derive qualitative characterizations of the dynamical systems. For the quantitative description of the dynamics, the small-scale patterns in the RP can be used, such as diagonal and vertical lines. The histograms of the lengths of these lines are the base of the recurrence quantification analysis (RQA)developed by Webber and Zbilut and later by Marwan et al. [7]. Based on the statistical properties of the recurrence plot, a large number of quantifiers have been developed to analyze details of a RP. Many of them, deal with statistical properties such as mean size, maximum size, frequency of occurrence of diagonal, vertical or horizontal recurrence lines.

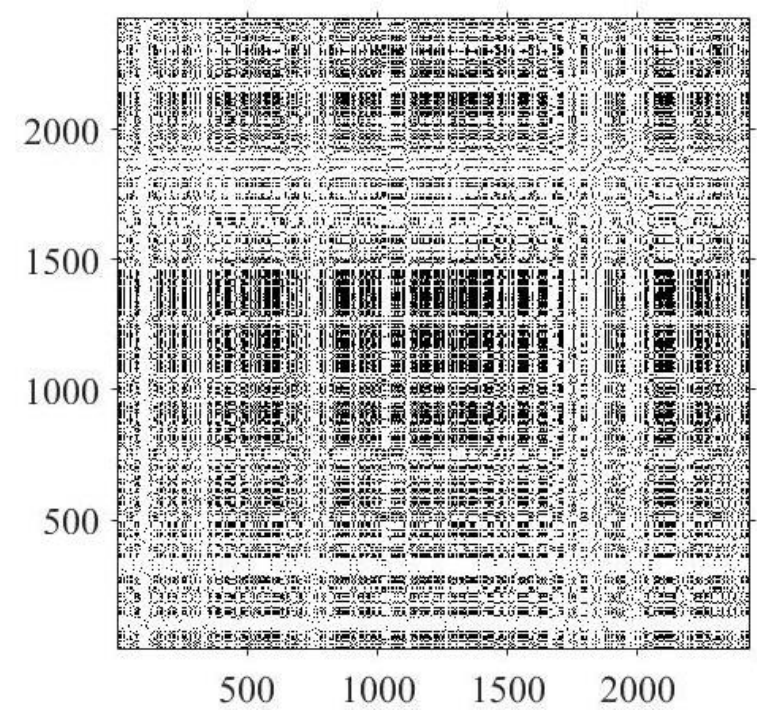

Fig. 2. Recurrence plot of daily values of BTC/\$ price fluctuations.

An important class of recurrence quantifiers are those that try to capture the level of complexity of a signal. As an example, we mention the already known entropy based on diagonal lines statistics. This quantity has been correlated with others dynamical quantifiers as, for example, the largest Lyapunov exponent, since both capture properties of the complexitylevel of the dynamics. The vertical (horizontal) lines in $R_{i j}$ are associated to laminar states, common in intermittent dynamics [7]. It was reported the use of the distribution of diagonal lines $P(l)$ for a different quantifier of recurrences, based on the Shannon entropy [7]. If we choose a distribution of diagonals $p(l)=P(l) / \sum_{l=1}^{K} P(l)$ for $K$ the maximum length of the diagonal lines, then we get one of the known quantitative indicators of recurrence analysis: $E N T R=-\sum_{l=l_{\min }}^{l=l_{\max }} p(l) \ln p(l)$. However, as follows from the analysis of entropy indicators, the results are not always possible to coordinate with the proposed models.

To the pleasure of the researchers, it turned out that depending on the technology of using the properties of the recurrence of the phase space, different types of recurrence entropies are distinguished [9].

\section{A. Recurrence probability (period) density entropy}

Recurrence probability (or period) density entropy (RPDEn) is useful for characterising the extent to which a time series repeats the same sequence $[10,11,19]$ and is, like the ENTR a quantitative characteristic of recurrence analysis. Around each point $x_{n}$ in the phase space, an $\varepsilon$ neighbourhood (an $m$-dimensional ball with this radius) is formed, and every time the time series returns to this ball, after having left it, the time difference $T$ between successive returns is recorded in a histogram. This histogram is normalised to sum to unity, to form an estimate of the recurrence period density function $P(T)$. The normalised entropy of this density $H_{n o r m}=-\left(\ln T_{\max }\right)^{-1} \sum_{t=1}^{T_{\max }} P(t) \ln P(t)$ is the RPDEn value, where $T_{\max }$ is the largest recurrence value.

\section{B. Recurrence entropy}

Recent works [12, 13] presents a slightly different technique for calculating recurrent entropy using a novel way to extract information from the recurrence matrix. The authors have generalize these concepts recurrence defining recurrence microstates $F(\varepsilon)$ as all possible cross-recurrence states among two randomly selected short sequences of $N$ consecutive points in a $K(K \square N)$ length time series, namely $F(\varepsilon)$ are $N \times N$ small binary matrices. The total number of microstates for a given $N$ is $N_{m s}=2^{N^{2}}$. The microstates are populated by $\bar{N}$ random samples obtained from the recurrence matrix such that $\bar{N}=\sum_{i=1}^{N_{m s}} n_{i}$, where $n_{i}$ is the number of times that a microstate $i$ is observed. For $P_{i}=n_{i} / \bar{N}$, the probability related to the microstate $i$, we define an entropy of the RP associated with the probabilities of occurrence of a microstate as $S\left(N_{m s}\right)=\sum_{i=1}^{N_{m s}} P_{i} \ln P_{i}$.

\section{RECURRENCE ENTROPY FOR CRASH TIME SERIES}

To study the recurrence entropy properties of time series, including periods of crisis, the following databases have been prepared. The first database included fragments of the Dow Jones index for the famous crashes of 1929, 1987, and 2008. In a number of daily values of the DJIA index of 2000 days long, the actual day of the onset of the crash falls at point 
1000 (Figure 3). In this case, the fixed point of crashes can easily observe the indicator capabilities of entropy measures of complexity.

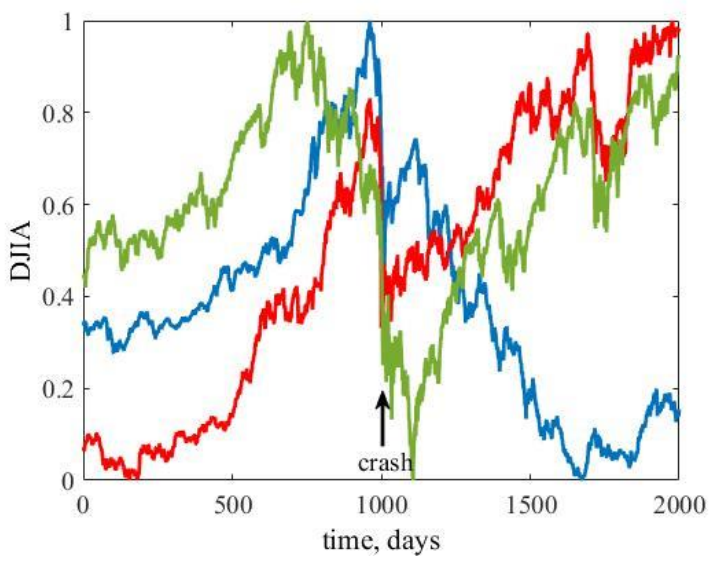

Fig. 3. Fragments of DJIA index with crash at 1000 days.

The following database contains the same length daily values (from March 3, 1990 to August 30, 2019) of the US stock market indices (DJIA), Germany (DAX), France $(\mathrm{CAC})$, used to check the universality of the complexity measure regardless of the index. The index DJIA is also taken for the period from January 1, 1983 to August 30, 2019 in order to cover the crises of 1987 and 1998.

The third database includes the values of daily Bitcoin prices for the entire observation period (from July 17, 2010) and for a shorter period of stabilization of the cryptocurrency market from January 1, 2013 to August 30, 2019.

Calculations of recurrent entropy measures of complexitywere carried out within the framework of amoving (sliding) window algorithm. For this purpose, the part of the time series (window), for whichthere were measures of complexity (RPDEn, RecEn), was selected, then the windowwas displaced along the time series in a one-day increment and the procedure repeateduntil all the studied series had exhausted. Further, comparing the dynamics of theactual time series and the corresponding measures of complexity, we can judge thecharacteristic changes in the dynamics of the behavior of complexity with changes inthe time series. If this or that measures of complexity behaves in a definite wayfor all periods of crisis, for example, decreases or increases during the pre-crisis period,then it can serve as an indicator or precursor of such a crisis phenomenon.

In Figure 4 presents the results of calculations RPDEn and RecEn for the first database with a length of 2000 days. The calculations were carried out for a sliding window size of 250 days and a step of 1 day. It can be seen from the figure that the recurrence entropy in the pre-crisis period is markedly reduced for all crisis events, which is obviously a precursor of such crisis phenomena. As for RPDEn, such an unambiguous precursor is not observed.

Therefore, further we focus on the use of RecEn, leaving for the future a more complete study of RPDEn.

In Figure 5 shows the RecEn dynamics for the long index DJIA, which includes the last seven well-known crashes (shown in the Figure).

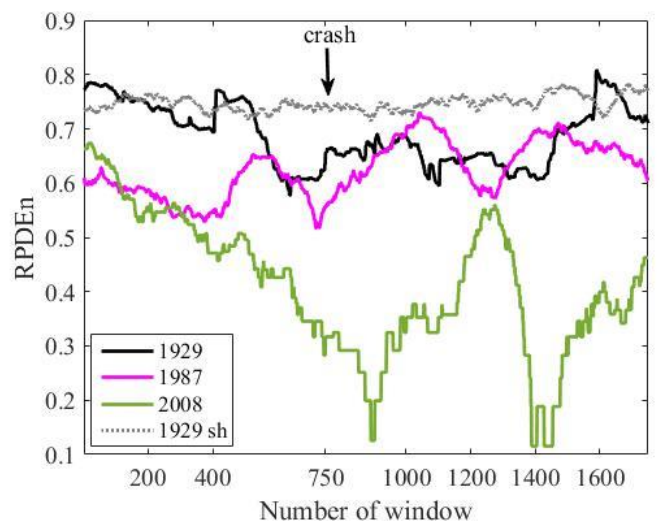

a)

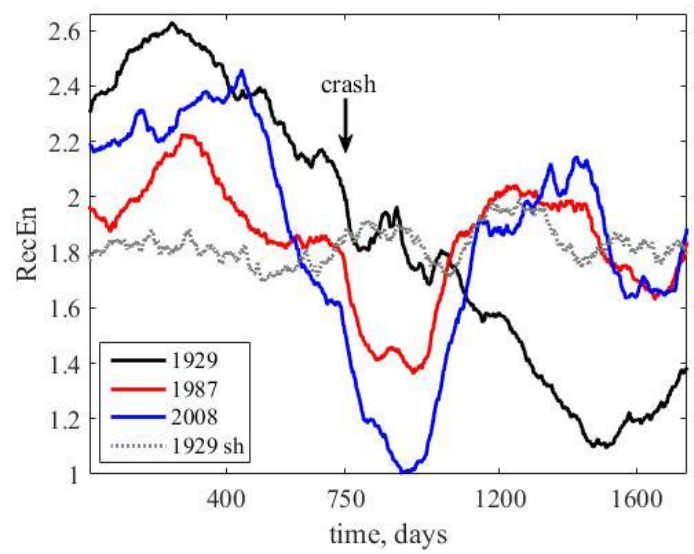

b)

Fig. 4. Window recurrence measures of complexity for the crashes of 1929, 1987, and 2008. a) RPDEn, b) RecEn. The start point of the crash is marked.

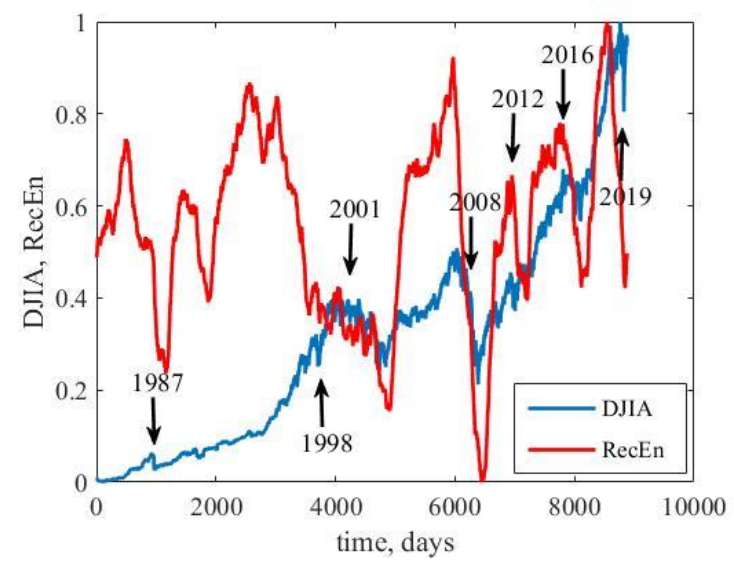

Fig. 5. Comparative dynamics of index DJIA and recurrence entropy RecEn.

Obviously, in this case, RecEn is the precursor of crash events in all these cases.

In order to once again verify the universality of RecEn as an indicator-precursor of financial crahes, we examined its dynamics for various stock indices. As an example, the selected indices are the stock markets of the USA (S\&P 500), Germany (DAX) and France (CAC) for a comparable period of time (Figure 6). 


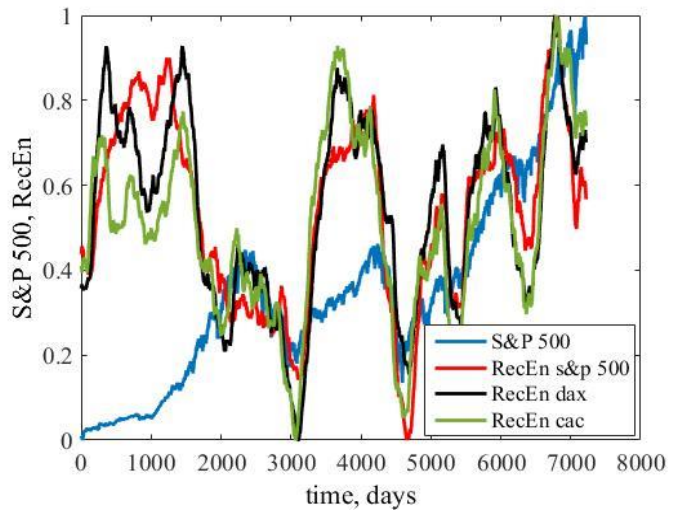

Fig. 6. The dynamics of the stock index S\&P 500 and its corresponding and also the indices of the DAX and CAC of recurrence entropy.

Finally, the analysis of a very volatile cryptocurrency market for BTC/\$ data with small window values (50 days) also allows us to identify the main crisis falls in this market (Figure 7).

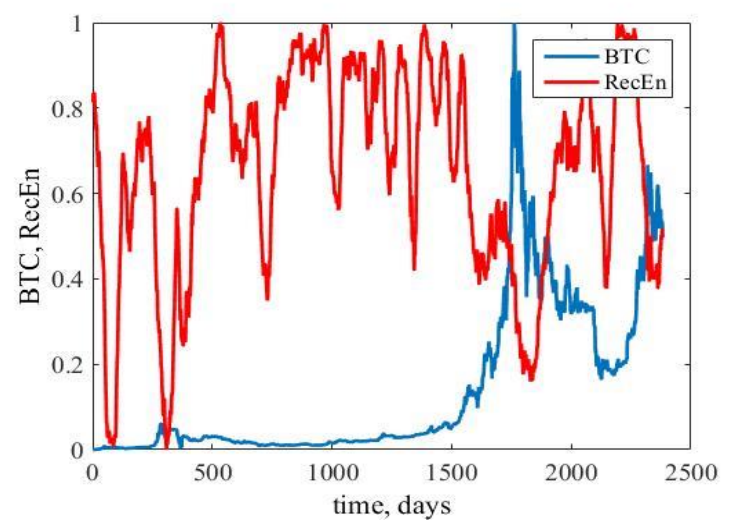

Fig. 7. Comparison of the dynamics of the BTC/\$ price with the corresponding recurrence entropy.

The periodization of bitcoin crises, which we conducted earlier, indicates that recurrent entropy in this case is also a harbinger of crisis phenomena.

\section{CONCLUSION}

Thus, we have demonstrated that the entropy analysis of financial time series in phase space reveals the characteristic recurrent properties of complex systems. It turned out that recurrent entropy, unlike other entropy indicators of complexity, is an indicator and an early harbinger of crisis phenomena. The recurrence entropy methodology has several advantages compared to the traditional recurrence entropy defined in the literature, namely, the correct evaluation of the chaoticity level of the signal, the weak dependence on parameters.

In the future, a thorough comparative analysis of the possibilities of recurrent entropy with other promising types of entropy indicators of complexity should be carried out [14 $-18]$.

\section{REFERENCES}

[1] D. Sornette, "Why Stock Markets Crash: Critical Events in Complex Systems". Princeton University Press, 2003.

[2] J. Diaz, "Evidence of Noisy Chaotic Dynamics in the Returns of Four Dow Jones Stock Indices. Annual Review of Chaos Theory", Bifurcation and Dynamical System, vol. 4, pp. 1-15, 2013.

[3] H. Kantz and T. Shreiber, "Nonlinear time series analysis, $2^{\text {nd }}$ ed"., Cambridge University Press, p. 369, 2004.

[4] V. Soloviev and A. Belinskij, "Complex Systems and Crashes of Cryptocurrency Market”, In: Ermolayev, V., Suárez-Fgueroa, M., Yakovyna, V., Mayr, H., Nikitchenko, M., Spivakovsky, A. (eds.) on ITC in Education, Research, and Industrial Applications. CCIS, vol 1007, pp 276-297. Springer, Cham, 2018.

[5] V. Soloviev and A. Belinskij, "Entropy analysis of crisis phenomena for DJIA index", In: Vadim Ermolayev, Frédéric Mallet, Vitaliy Yakovyna, Vyacheslav Kharchenko, Vitaliy Kobets, Artur Korniłowicz, Hennadiy Kravtsov, Mykola Nikitchenko, Serhiy Semerikov, Aleksander Spivakovsky (eds.) on ITC in Education, Research, and Industrial Applications, 2019.

[6] A. Belinskyi, V. Soloviev, S. Semerikov and V. Solovieva, "Detecting stock crashes using Levy distribution", CEUR-WS, vol. 2422, pp. 420-433, 2019.

[7] N. Marwan, N., Romano, M.C, M. Thiel, M. and J. Kurths, "Recurrence plots for the analysis of complex systems", Phys. Rep., vol. 438, pp. 237-329, 2007.

[8] R. Zhou, R. Cai and G. Tong, "Applications of entropy in finance: a review”, Entropy, vol. 15, pp. 4909-4931, 11 Nov. 2013.

[9] P. Faure and A. Lesne, "Estimating Kolmogorov entropy from recurrence plots", in Recurrence Quantification Analysis, Gr. Ch. L Webber and N. Marwan, Eds. Springer International Publishing, 2015, pp. 45-64.

[10] H. Rabarimanantsoa, L. Achour, C. Letellier, A. Cuvelier and J.-F. Muir, "Recurrence plots and Shannon entropy for a dynamical analysisof asynchronisms in noninvasive mechanical ventilation", Chaos, vol. 17, 013115, 21 Mar. 2007.

[11] M. A. Little, P. E. McSharry, S. J. Roberts, D. AE. Costello, and I. M. Moroz, "Exploiting nonlinear recurrence and fractal scaling properties forvoice disorder detection", BioMedical Engineering OnLine, vol. 6, pp. 1-19, 26 Jun. 2007.

[12] G. Corso, T. Prado, G. Lima and S. Lopes, "A novel entropy recurrence quantification analysis", arXiv:1707.00944v1 [stat.OT] 4 Jul. 2017.

[13] S. Lopes, T. Lima, G. Corso, G. Lima and J. Kurths, "Parameter-free quantification of stochastic and chaotic signals", arXiv: 1905.02284v1 [physics.data-au] 6 May 2019.

[14] H. Danylchuk, V. Derbentsev, V. Soloviev, and A. Sharapov, "Entropy analysis of dynamics properties of regional stock market", Science and Education a New Dimension. Economics, vol. 4(2), pp. 15-19, 2016.

[15] T. Pele, E. Lazar and A. Dufour, "Information Entropy and Measures of Market Risk”, Entropy,vol. 19(5), pp. 1-19, 2017

[16] R. Lim, "Rapid Evaluation of Permutation Entropy for Financial Volatility Analysis - A Novel Hash Function using Feature-Bias Divergence". Department of Computer Science, Imperial College of London, London, 2014.

[17] R. Gu, "Multiscale Shannon Entropy and its application in the stock market", Physics A, vol. 484, pp. 215-224, 2017.

[18] G.-J. Wang, C. Xie and F. Han, "Multi-Scale Approximation Entropy Analysis of Foreign Exchange Markets Efficiency". Systems Engineering Procedia, vol. 3, pp. 201-208, 2012.

[19] https://en.wikipedia.org/wiki/Recurrence_period_density_entropy 